\title{
A PROOF OF BRAUER'S THEOREM ON GENERALIZED DECOMPOSITION NUMBERS
}

\author{
HIROSI NAGAO
}

To Professor Richard Brauer on the occasion of his 60th birthday

In [3] $\mathrm{R}$. Brauer gave a proof of his theorem on generalized decomposition numbers which was first announced in [1], and a simplification of it has been made by K. Iizuka [5]. In this note we shall show that the theorem may be proved from another point of view by using some results obtained by J. A. Green in [4].

After stating some results by Green and Osima in the first and second sections we first prove a theorem on characters (Theorem 1) and by using the theorem we prove Brauer's theorem in the fourth section.

\section{The algebra $Z(\$ 3:$ S)}

Let $\mathbb{B}$ be a finite group. We consider the group ring $I(\mathbb{S})$ of $\mathbb{B}$ over the ring $\mathfrak{D}$ of $\mathfrak{p}$-adic integers, where $\mathfrak{p}$ is a prime ideal divisor of a fixed prime $p$ in some algebraic number field.

If $G$ is any element of $\$\left(Y, r\right.$ any element of $\Gamma(\$)$, write $\gamma^{G}=G^{-1} \gamma G$. Then for a subgroup $\mathfrak{S}$ of $\mathbb{B}$ the set

$$
Z(\mathbb{S}: \mathfrak{S})=\left\{\gamma \in \Gamma(\mathbb{S}): \gamma^{H}=\gamma \text { for all } H \in \mathfrak{S}\right\}
$$

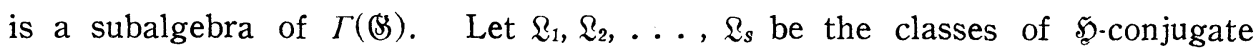
elements in $\mathbb{B}$, where two elements $X$ and $Y$ of $\mathbb{B}$ are called $\mathfrak{S}$-conjugate if there exists an element $H$ in $\mathfrak{S}$ such that $Y=X^{H}$. If $L_{1}, L_{2}, \ldots, L_{s}$ denote the sums of the elements in $\mathfrak{I}_{1}, \mathfrak{Z}_{2}, \ldots, \mathfrak{Q}_{s}$ respectively, these sums form an 0 basis of $Z(\mathbb{S}: \mathfrak{S})$.

For a fixed $\mathfrak{S}$-conjugacy class $\mathfrak{L}_{\alpha}$, a Sylow $p$-subgroup of the normalizer $\Re_{\mathfrak{S}}\left(U_{\alpha}\right)$ of some element $U_{\alpha} \in \mathfrak{L}_{\alpha}$ in $\mathfrak{S}_{2}$ is called the $p$-defect group of $\mathfrak{L}_{\alpha}$, and is

Received by Journal of Mathematics, Osaka City Univereity, December 13, 1962; Transfered to Nagoya Mathematical Jounal January 29, 1963. 
denoted by $\mathfrak{P}_{\alpha}$. It is determined up to $\mathfrak{S}$-conjugacy.

Let $\mathfrak{P}$ be a $p$-subgroup of $\mathfrak{S}$ and $I(\mathfrak{P})$ the set of those $\alpha \in\{1,2, \ldots, s\}$ such that $\mathfrak{P}_{\alpha} \leq \mathfrak{F}$, i.e. $\mathfrak{P}_{\alpha} \leq H^{-1} \mathfrak{P} H$ for some $H \in \mathfrak{S}$. The set of all $z \in Z(\mathbb{B}: \mathfrak{F})$ of the form

$$
z \equiv \sum_{\alpha \in I(\mathfrak{B})} a_{\alpha} L_{\alpha} \bmod \mathfrak{p} Z(\mathfrak{B}: \mathfrak{S}) \quad\left(a_{\alpha} \in \mathfrak{p}\right)
$$

is denoted by $Z_{\mathfrak{S}}(\stackrel{\Re}{(\mathfrak{S}})$.

Lemma 1 (Osima [6], Green [4], Lemma $3.2 \mathrm{c}$ ). If $\mathfrak{P}$ is a p-subgroup of $\mathfrak{g}$, then $Z_{\mathfrak{P}}(\mathfrak{S}: \mathfrak{S})$ is an ideal of $Z(\mathbb{S}: \mathfrak{H})$.

\section{Characters}

If a right $\Gamma(\$)$-module $M$ is free and finitely generated over $\mathfrak{D}$ and unitary, i.e. $m 1=m$ for all $m \in M$, we call $M$ a representation module of $\mathbb{B}$ over $\mathfrak{D}$ or \$-representation module for short. A \&-representation module $M$ has an Dbasis, and hence a matrix representation is associated with $M$. The character of the matrix representation associated with $M$ is denoted by $\%_{M}$.

A $\mathbb{3}$-representation module $M$ is said to be $\mathfrak{5}$-projective if $M$ is a direct summand of the induced module $N \otimes_{\Gamma(\mathfrak{F})} \Gamma(\mathbb{S})$ of some $\mathfrak{S}$-representation module $N$.

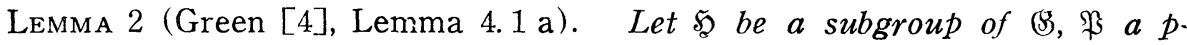
subgroup of $\mathfrak{S}$ and let $M$ be a $\mathbb{S}$-representation module. If $e$ is an idempotent in $Z_{\mathfrak{B}}(\mathfrak{S}: \mathfrak{S})$, then $\mathfrak{5}$-representation module $M e$ is $\mathfrak{P}$-projective.

If for an element $X$ of $\& X=P V=V P$, where $P$ has order a power of $p$ and $V$ has order prime to $p, P$ and $V$ are called $p$-factor and $p$-regular factor of $X$, respectively. The following is one of the main theorems by Green in [4].

Lemma 3 (Green [4], Theorem 3). Let is be a p-subgroup of $\mathbb{B}$ and $M a$ (B-representation module. If $M$ is $\mathfrak{P}$-projective and the p-factor of an element $X$ does not lie in any conjugate of $\mathfrak{P}$, then

$$
\chi_{M}(X)=0
$$

\section{Brauer homomorphisms}

Let $\mathfrak{F}$ be a given $p$-subgroup of $\mathbb{B}$ and let $\mathscr{S}$ be a subgroup such that $\mathfrak{P C}(\mathfrak{F}) \leq \mathscr{S} \leq \mathfrak{M}(\mathfrak{P})$, where $\mathfrak{S}(\mathfrak{B})$ and $\mathfrak{N}(\mathfrak{P})$ are the centralizer and normalizer 


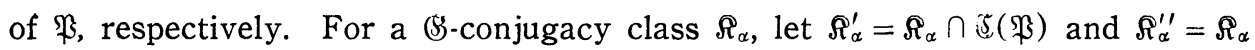
- $\Re_{\alpha}^{\prime}$. Denote by $K_{\alpha}^{\prime}, K_{\alpha}^{\prime \prime}$ the sums of the elements in $\Re_{\alpha}^{\prime}, \mathcal{R}_{\alpha}^{\prime \prime}$, respectively. Then $\mathscr{R}_{\alpha}^{\prime}$ and $\mathscr{R}_{\alpha}^{\prime \prime}$ are collections of $\mathfrak{g}$-conjugacy classes, and hence $K_{\alpha}^{\prime}$ and $K_{\alpha}^{\prime \prime}$ are in $Z(\mathfrak{S}: \mathfrak{S})$. Each $\mathfrak{H}$-conjugacy class in $\mathscr{\Re}_{\alpha}^{\prime \prime}$ has the defect group $\mathfrak{Q}$ such that $\mathfrak{P} \$ \mathfrak{D}$.

Let $Z(\$)$ be the center of $T(\$)$ and $Z^{*}(\$)$ the residue algebra $Z(\$) / p Z(\mathbb{B})$. Then Brauer [2] has shown that the linear mapping $s^{*}: Z^{*}(\mathbb{B}) \rightarrow Z^{*}(\mathfrak{S})$ which is defined by $s^{*}\left(K_{\alpha}\right)=K_{\alpha}^{\prime}$ is an algebra homomorphism. We shall call this the Brauer homomorphism.

Let $E$ be an idempotent in $Z(\$)$ and $E^{*}$ the image of $E$ under the natural mapping $Z(\$) \rightarrow Z^{*}(\mathbb{S})$. As is well known, the idempotent $s^{*}\left(E^{*}\right)$ in $Z^{*}(\mathfrak{S})$ can be lifted to an idempotent $e$ of $Z(\mathscr{2})$, i.e. $e^{*}=s^{*}\left(E^{*}\right)$. Now, we consider the situation where $\mathfrak{P}$ is the cyclic subgroup generated by an element $P$ of order a power of $\boldsymbol{p}$ and $\mathfrak{S}$ is the centralizer $\mathfrak{S}(\mathfrak{P})=\mathfrak{R}(P)$ of $\mathfrak{\Re}$. Then we have

TheOREM 1. Let $P$ be an element of order a power of $p, E$ an idempotent of $Z(\$)$ and let $e$ be the idempotent of $Z(\Re(P))$ such that $s^{*}\left(E^{*}\right)=e^{*}$, where $s^{*}: Z^{*}(\$) \rightarrow Z^{*}(\Re(P))$ is the Brauer homomorphism. If $M$ is a (S)-representation module such that $M E=M$, then for any pregular element $V$ in $\Re(P)$, we have

$$
\chi_{M}(P V)=\chi_{M e}(P V) .
$$

Proof. If $E=\sum_{\alpha} b_{\alpha} K_{\alpha}$ then

$$
\boldsymbol{e} \equiv \sum_{\alpha} b_{\alpha} K_{\alpha}^{\prime} \quad \bmod \mathfrak{p} Z(\mathbb{B}: \mathfrak{R}(P))
$$

therefore

$$
E-e \equiv \sum_{\alpha} b_{\alpha} K_{\alpha}^{\prime \prime} \quad \bmod \mathfrak{p} Z(\&: \Re(P)) .
$$

Since each $\Re(P)$-conjugacy class in $\mathfrak{R}_{\alpha}^{\prime \prime}$ has the defect group $\mathfrak{Q}$ such that $P \notin \mathfrak{Q}$, $E-e$ lies in the ideal

$$
\Lambda=\sum_{P \notin \mathbb{Q}} Z_{\mathfrak{Q}}(\mathbb{S}: \Re(P))
$$

of $Z(\mathbb{B}: \Re(P))$, where the sum is over all $p$-subgroups $\mathscr{Q}$ of $\Re(P)$ which do not contain $P$. Let $f=E(E-e)$. Then $f \in \Lambda$, and $E e$ and $f$ are mutually orthogonal idempotents such that $E=E e+f$. Since $\mathrm{E} e$ and $f$ commute with all elements of $\Re(P), M E e$ and $M f$ are $\Re(P)$-representation modules. By the 
assumption $M E=M$, therefore $M$ is the direct sum of two $\Re(P)$-submodules $M E e=M e$ and $M f$

$$
M=M e \oplus M f
$$

Let $f=\sum f_{i}$, where $\left\{f_{i}\right\}$ is a set of mutually orthogonal primitive idempotents in $Z(\mathbb{S}: \Re(P))$. Since $f_{i}=f_{i} f \in \Lambda$, by a theorem of Rosenberg (cf. Green [4], Lemma 3.3 a) there is a $p$-subgroup $\mathfrak{Q}_{i}$ of $\mathfrak{N}(P)$ such that $P \notin \mathfrak{Q}_{i}$ and $f_{i} \in$ $Z \mathfrak{\beth}_{\imath}(\&: \Re(P))$, and then $M f_{i}$ is $\mathfrak{Q}_{i}$-projective by Lemma 2. For any $p$-regular element $V$ of $\mathfrak{l}(P)$, the $p$-factor of $P V$ is $P$ and $P$ does not lie in any subgroup $\mathfrak{N}(P)$-conjugate to $\mathfrak{Q}_{i}$, therefore by Lemma $3 \chi_{M f_{i}}(P V)=0$. Since

$$
M f=M f_{1} \oplus \cdots \oplus M f_{r},
$$

$\chi_{M f}(P V)=0$, and hence $\chi_{M}(P V)=\chi_{M e}(P V)$.

\section{Proof of Brauer's theorem}

Let $\left\{\chi_{i}\right\}$ be the set of absolutely irreducible ordinary characters of $B, P$ an element of order a power of $p$ and let $\left\{\tilde{\varphi}_{j}\right\}$ be the set of absolutely irreducible ordinary characters of $\Re(P)$. Let

$$
\chi_{i} \mid \Re(P)=\sum_{j} r_{i j} \tilde{\chi}_{j}
$$

be the decomposition of the restriction of $\gamma_{i}$ to $\Re(P)$, and let

$$
\tilde{\chi}_{j}=\sum_{\mu} \tilde{d}_{j \mu} \widetilde{\varphi}_{\mu}
$$

be the $p$-modular decomposition of $\tilde{\chi}_{j}$, where the $\tilde{\varphi}_{\mu}$ are the irreducible $p$ modular characters of $\Re(P)$ and the $\widetilde{d}_{j \mu}$ are the decomposition numbers of $\Re(P)$. Since $P$ is in the center of $\Re(P)$

$$
\tilde{\chi}_{j}(P V)=\varepsilon_{j} \tilde{\chi}_{j}(V)=\sum_{\mu} \varepsilon_{j} \tilde{d}_{j \mu} \tilde{\varphi}_{\mu}(V)
$$

for any $p$-regular element $V$ in $\Re(P)$, where $\varepsilon_{j}=\frac{\tilde{\chi}_{j}(P)}{\widetilde{\chi}_{j}(1)} \cdot$ From (1), (2) and

$$
\chi_{i}(P V)=\sum_{\mu} d_{i \mu}^{P} \tilde{\varphi}_{\mu}(V)
$$

for any $p$-regular element $V$ of $\Re(P)$, where $d_{i \mu}^{P}=\sum_{j} r_{i j} \varepsilon_{j} \widetilde{d}_{j \mu}$. The $d_{i^{k}}^{P}$ are called the generalized decomposition numbers of $\$$.

Now suppose that $D$ contains a primitive $g$-th root of unity, where $g$ is the 
order of $\$$. Let $E$ be a primitive idempotent of $Z(B)$. Any $\%_{i}$ is the character of some representation module $M_{i}$ of $\mathbb{B}$ over $\mathfrak{B}$. If $M_{i} E=M_{i}$ then we say that $\chi_{i}$ belongs to the $p$-block $B$ associated with $E$.

Let $e$ be the idempotent in $Z(\Re(P))$ such that $e^{*}=s^{*}\left(E^{*}\right)$, where $s^{*}: Z^{*}(\mathbb{S})$ $\rightarrow Z^{*}(\mathfrak{R}(P))$ is the Brauer homomorphism. If $\widehat{B}$ is the set of $\tilde{\chi}_{j}$ such that the associated representation medule $\tilde{M}_{j}$ of $\Re(P)$ over $\mathfrak{D}$ satisfies $\tilde{M}_{j} e=\tilde{M}_{j}$, then $\tilde{B}$ is a collection of $p$-blocks of $\Re(P)$. We shall also denote by $\widehat{B}$ the set of $p$ modular characters $\widetilde{\varphi}_{\mu}$ of $\Re(P)$ such that $\tilde{d}_{j \mu} \neq 0$ for some $\tilde{\chi}_{j} \in \widetilde{B}$. Then the Brauer's theorem reads as follows:

TheOREM 2. If $\chi_{i}$ belongs to a p-block $B$ of $\mathbb{B}$, then the generalized decomposition numbers $d_{i \mu}^{P}$ can be different from zero only for $\widetilde{\varphi}_{\mu}$ which belongs to $\widetilde{B}$.

Proof. Let $V$ be any $p$-regular element of $\Re(P)$. Let

$$
\chi_{i}=\sum_{j}^{\prime} r_{i j} \tilde{\chi}_{j}+\sum_{k}^{\prime \prime} r_{i k} \tilde{\chi}_{k}
$$

where the sum $\Sigma^{\prime}$ is over all $\tilde{\chi}_{j}$ in $\widetilde{B}$ and the sum $\Sigma^{\prime \prime}$ is over all other $\tilde{\chi}_{k}$. Then from Theorem 1 we have

$$
\begin{aligned}
\gamma_{i}(P V) & =\sum_{j}^{\prime} r_{i j} \tilde{\chi}_{j}(P V) \\
& =\sum_{\mu}{ }^{\prime} d_{i \mu}^{P} \tilde{\varphi}_{\mu}(V),
\end{aligned}
$$

where $\mu$ ranges over the suffices such that $\widetilde{\varphi}_{\mu} \in \widetilde{B}$. Since the $\tilde{\varphi}_{\mu}$ are linearly independent, we have the therem.

\section{REFERENCES}

[1] R. Brauer, On blocks of characters of groups of finite order, II, Proc. Nat. Acad. Sci. U.S.A. 32, 215-219 (1946).

[2] R. Brauer, Zur Darstellunstheorie der Gruppen endlicher Ordnung, Math. Z. 63, 406444 (1956).

[3] R. Brauer, Zur Darstellugstherie der Gruppen endlicher Ordnung, II, Math. Z. 72, 25-46 (1959).

[4] J. A. Green, Brocks of modular representations, Math. Z. 79, 100-115 (1962).

[5] K. Iizuka, On Brauer's theorem on sections in the theory of blocks of group characters, Math. Z. 75, 299-304 (1961).

[6] M. Osima, Notes on blocks of group characters, Math. J. Okayama Univ. 4, 175-188 (1955).

\section{Osaka City University}

